\title{
pulver: an R package for parallel ultra-rapid $p$-value computation for linear regression interaction terms
}

\author{
Sophie Molnos ${ }^{1,2,3^{*}}$ (D), Clemens Baumbach ${ }^{1,2,3}$, Simone Wahl ${ }^{1,2,3}$, Martina Müller-Nurasyid ${ }^{4,5,6,7}$, \\ Konstantin Strauch ${ }^{5,6}$, Rui Wang-Sattler ${ }^{1,2}$, Melanie Waldenberger ${ }^{1,2}$, Thomas Meitinger ${ }^{8,9}$, Jerzy Adamski ${ }^{3,10,11}$, \\ Gabi Kastenmüller $^{12,13}$, Karsten Suhre ${ }^{12,14}$, Annette Peters ${ }^{1,2,3}$, Harald Grallert ${ }^{1,2,3}$, Fabian J. Theis ${ }^{15,16}$ and \\ Christian Gieger ${ }^{1,2,3}$
}

\begin{abstract}
Background: Genome-wide association studies allow us to understand the genetics of complex diseases. Human metabolism provides information about the disease-causing mechanisms, so it is usual to investigate the associations between genetic variants and metabolite levels. However, only considering genetic variants and their effects on one trait ignores the possible interplay between different "omics" layers. Existing tools only consider single-nucleotide polymorphism (SNP)-SNP interactions, and no practical tool is available for large-scale investigations of the interactions between pairs of arbitrary quantitative variables.

Results: We developed an R package called pulver to compute $p$-values for the interaction term in a very large number of linear regression models. Comparisons based on simulated data showed that pulver is much faster than the existing tools. This is achieved by using the correlation coefficient to test the null-hypothesis, which avoids the costly computation of inversions. Additional tricks are a rearrangement of the order, when iterating through the different "omics" layers, and implementing this algorithm in the fast programming language $\mathrm{C}++$. Furthermore, we applied our algorithm to data from the German KORA study to investigate a real-world problem involving the interplay among DNA methylation, genetic variants, and metabolite levels.

Conclusions: The pulver package is a convenient and rapid tool for screening huge numbers of linear regression models for significant interaction terms in arbitrary pairs of quantitative variables. pulver is written in $\mathrm{R}$ and $\mathrm{C}++$, and can be downloaded freely from CRAN at https://cran.r-project.org/web/packages/pulver/.
\end{abstract}

Keywords: Algorithm, Linear regression interaction term, SNP-CpG interaction, Software

\section{Background}

Hundreds of genetic variants associated with complex human diseases and traits have been identified by genome-wide association studies (GWAS) [1-4]. However, most GWAS only considered univariate models with one outcome and one independent variable, thereby ignoring possible interactions between different quantitative "omics" data [5], such as DNA methylation,

\footnotetext{
* Correspondence: Sophie.molnos@helmholtz-muenchen.de

${ }^{1}$ Research Unit of Molecular Epidemiology, Helmholtz Zentrum München, Neuherberg, Germany

${ }^{2}$ Institute of Epidemiology II, Helmholtz Zentrum München, Neuherberg, Germany

Full list of author information is available at the end of the article
}

genetic variations, mRNA levels, or protein levels. For example, studies observed associations between specific epigenetic-genetic interactions and a phenotype [6-8]. The lack of publications analyzing genome-wide interactions may result because of the high computational cost of running linear regressions for all possible pairs of "omics" data. Understanding the interplay among different "omics" layers can provide important insights into biological pathways that underlie health and disease [9].

Previous interaction analyses in genome-wide studies mainly considered interactions between single-nucleotide polymorphisms (SNPs), which led to the development of several rapid analysis tools. For example, BiForce [10] is a 
stand-alone Java program that integrates bitwise computing with multithreaded parallelization; SPHINX [11] is a framework for genome-wide association mapping that finds SNPs and SNP-SNP interactions using a piecewise linear model; and epiGPU [12] calculates contingency table-based approximate tests using consumer-level graphics cards.

Several rapid programs are also available for calculating linear regressions without interaction terms. For example, OmicABEL [13] efficiently exploits the structure of the data but does not allow the inclusion of an interaction term. The $\mathrm{R}$ package MatrixEQTL [14] computes linear regressions very quickly based on matrix operations. This package also allows for testing for interaction between a set of independent variables and one fixed covariate. However, interactions between arbitrary pairs of quantitative covariates would require iteration over covariates, which is quite inefficient.

Thus, our R package called pulver is the first tool to allow the user to compute $p$-values for interaction terms in huge numbers of linear regressions in a practical amount of time. The acronym pulver denotes parallel ultra-rapid $p$-value computation for linear regression interaction terms.

We benchmarked the performance of our implemented method using simulated data. Furthermore, we applied our algorithm to "omics" data from the Cooperative Health Research in the Region of Augsburg (KORA) F4 study (DNA methylation, genetic variants, and metabolite levels).

KORA comprises a series of independent populationbased epidemiological surveys and follow-up studies of participants living in the region of Augsburg, Southern Germany [15].

Access to the KORA data can be requested via the KORA.Passt System (https://helmholtz-muenchen.managed-otrs.com/otrs/customer.pl).

\section{Implementation}

pulver computes $p$-values for the interaction term in a series of multiple linear regression models defined by covariate matrices $X$ and $Z$ and an outcome matrix $Y$, containing continuous data, e.g. metabolite levels, mRNA or proteomics data. In most cases the residuals from the phenotype adjusted for other parameters are used. All matrices must have equal number of rows, i.e., observations. For efficiency reasons, pulver does not adjust for additional covariates, instead the residuals from the phenotype adjusted for other parameters should be used.

\section{Linear regression analysis}

For every combination of columns $x, y$, and $z$ from matrices $X, Y$, and $Z$, pulver fits the following multiple linear regression model:

$$
y=\beta_{0}+\beta_{1} x+\beta_{2} z+\beta_{3} x z+\varepsilon, \varepsilon^{\sim} \text { i.i.d.N }\left(0, \sigma^{2}\right),
$$

where $y$ is the outcome variable, $x$ and $z$ are covariates, and $x z$ is the interaction (product) of covariates $x$ and $z$. All variables are quantitative. We need to test the null hypothesis $\beta_{3}=0$ against the alternative hypothesis $\beta_{3} \neq$ 0 . In particular, we are not interested in estimating the coefficients $\beta_{1}$ and $\beta_{2}$, which allows us to take a computational shortcut. By centering and orthogonalizing the variables, we can reduce the multiple linear regression problem into a simple linear regression without intercept. Thus, we can compute the Student's $t$-test statistic for the coefficient $\beta_{3}$ as a function of the Pearson's correlation coefficient between $y$ and the orthogonalized $x z: t=r \sqrt{D F /\left(1-r^{2}\right)}$, where $D F$ is the degree of freedom. See the Additional file 1 for a more detailed derivation.

By computing the $t$-statistic based on the correlation coefficient, which has a very simple expression in the simplified model, we avoid fitting the entire model including estimating the coefficients $\beta_{1}$ and $\beta_{2}$. This is much more efficient because we are actually only interested in the interaction term.

\section{Avoiding redundant computations}

Despite the computational shortcut, even more time can be saved by employing a sophisticated arrangement of the computations. The naïve approach would iterate through three nested for-loops, with one for each matrix, where all computations occur in the innermost loop. However, Fig. 1 shows that some computations can be moved out of the innermost loop to avoid redundant computations.

\section{Programming language and general information about the program}

We implemented the algorithm in an $\mathrm{R}$ package [16] called pulver. Due to speed considerations, the core of the algorithm was implemented in $\mathrm{C}++$. We used $\mathrm{R}$ version 3.3.1 and compiled the $\mathrm{C}++$ code with gcc compiler version 4.4.7. To integrate $\mathrm{C}++$ into $\mathrm{R}$, we used the $\mathrm{R}$ package Rcpp [17] (version 0.12.7).

To determine whether $\mathrm{C} /$ Fortran could improve the performance compared to that of $\mathrm{C}++$, we also implemented the algorithm using a combination of $\mathrm{C}$ and Fortran via R's C interface.

We used OpenMP version 3.0 [18] to parallelize the middle loop. To minimize the amount of time required to coordinate parallel tasks, we inverted the order of matrices $X$ and $Z$ so that the middle loop could run over more variables than the outer loop, thereby maximizing the amount of work per thread.

To improve efficiency, the program does not allow covariates other than $x$ and $z$. If additional covariates are required, the outcome $y$ must be replaced by the residuals from the regression of $y$ on the additional covariates. 


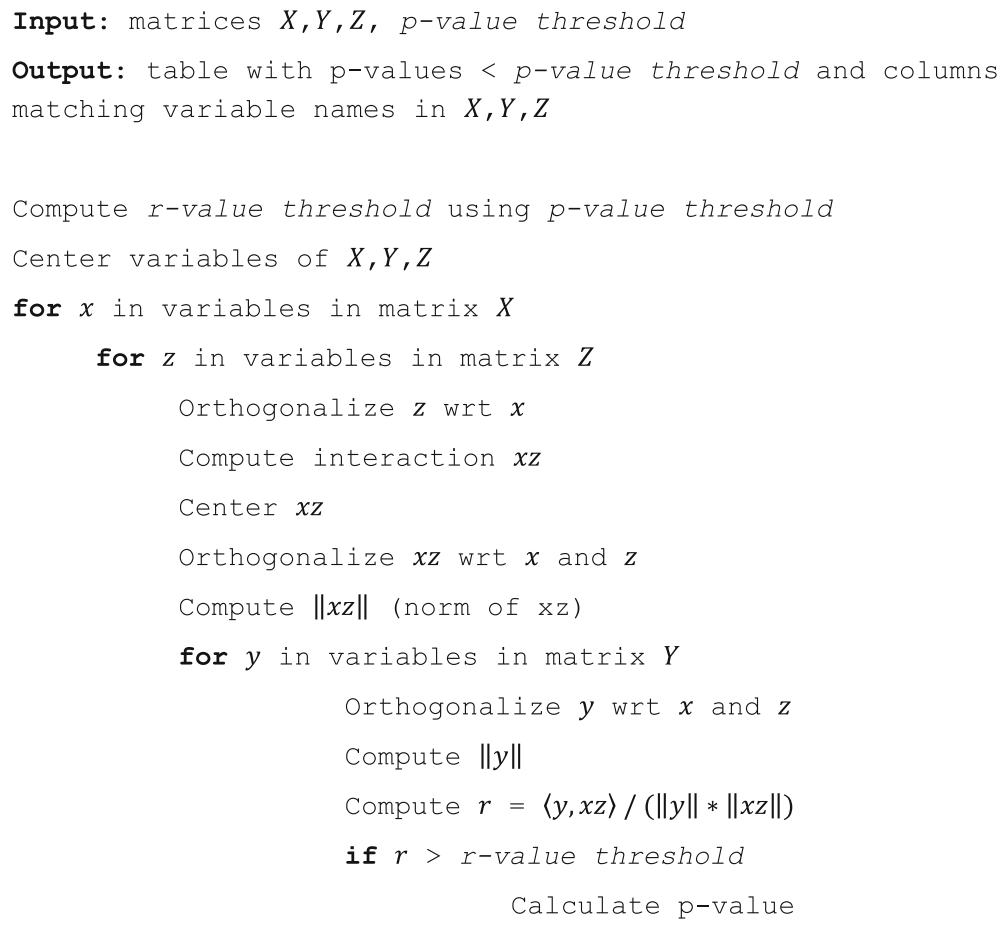

Fig. 1 Pseudo-code of the pulverize function

Missing values in the input matrices are replaced by the respective column mean.

Our pulver package can be used as a screening tool for scenarios where the number of models (number of variables in matrix $X \times$ number of variables in matrix $Y \times$ number of variables in matrix $Z$ ) is too large for conventional tools. By specifying a $p$-value threshold, the results can be limited to models with interaction term $p$-values below the threshold, thereby reducing the size of the output greatly. After the initial screening process, additional model characteristics for the significant models, e.g., effect estimates and standard errors, can be obtained with traditional methods such as R's $l m$ function.

The user can access pulver's functionality via two functions: pulverize and pulverize_all. The pulverize function expects three numeric matrices and returns a table with p-values for models with interaction term $p$-values below the (optionally specified) $p$-value threshold. The wrapper function pulverize_all expects files with names containing $X, Y$, and $Z$ matrices, calls pulverize to perform the actual computation, and returns a table in the same format as pulverize. The pulverize_all function is particularly useful if the matrices are too huge to be loaded all at the same time because of the computer memory restrictions. Thus, pulverize_all gets inputs as lists of file names containing the submatrices $\mathrm{X}, \mathrm{Y}$, and $\mathrm{Z}$. pulveri$z e$ all iterates through these lists and subsequently loads matrices before calling the pulverize.

\section{Comparisons with other $\mathbf{R}$ tools for running linear regressions}

As illustrated in Fig. 2, the inputs for the interaction analysis can be vectors or matrices. Compared to other $\mathrm{R}$ tools such as $\mathrm{lm}$ and MatrixEQTL pulver is currently the only available option for users who want all the inputs to be matrices. It is possible to adapt other tools to all-matrix inputs, but the resulting code is not optimized for this use and will be too slow for practical purposes.

$$
p_{1}, p_{2} \text { and } p_{3} \text { are } \in \mathbb{N} \text {. }
$$

\section{Results}

To benchmark the performance of pulver against other tools, we simulated $X, Y$, and $Z$ matrices with different numbers of observations and variables.

We also applied pulver to real data from the KORA study.

\section{Performance comparison using simulated data}

No other tool is specialized for the type of interaction analysis described above, so we compared the speed of our $\mathrm{R}$ package pulver with that of R's built-in lm function and the $\mathrm{R}$ package MatrixEQTL [14] (version 2.1.1) (also see Fig. 2).

To ensure a fair comparison, we did not use the parallelization feature of pulverize because it is not available 


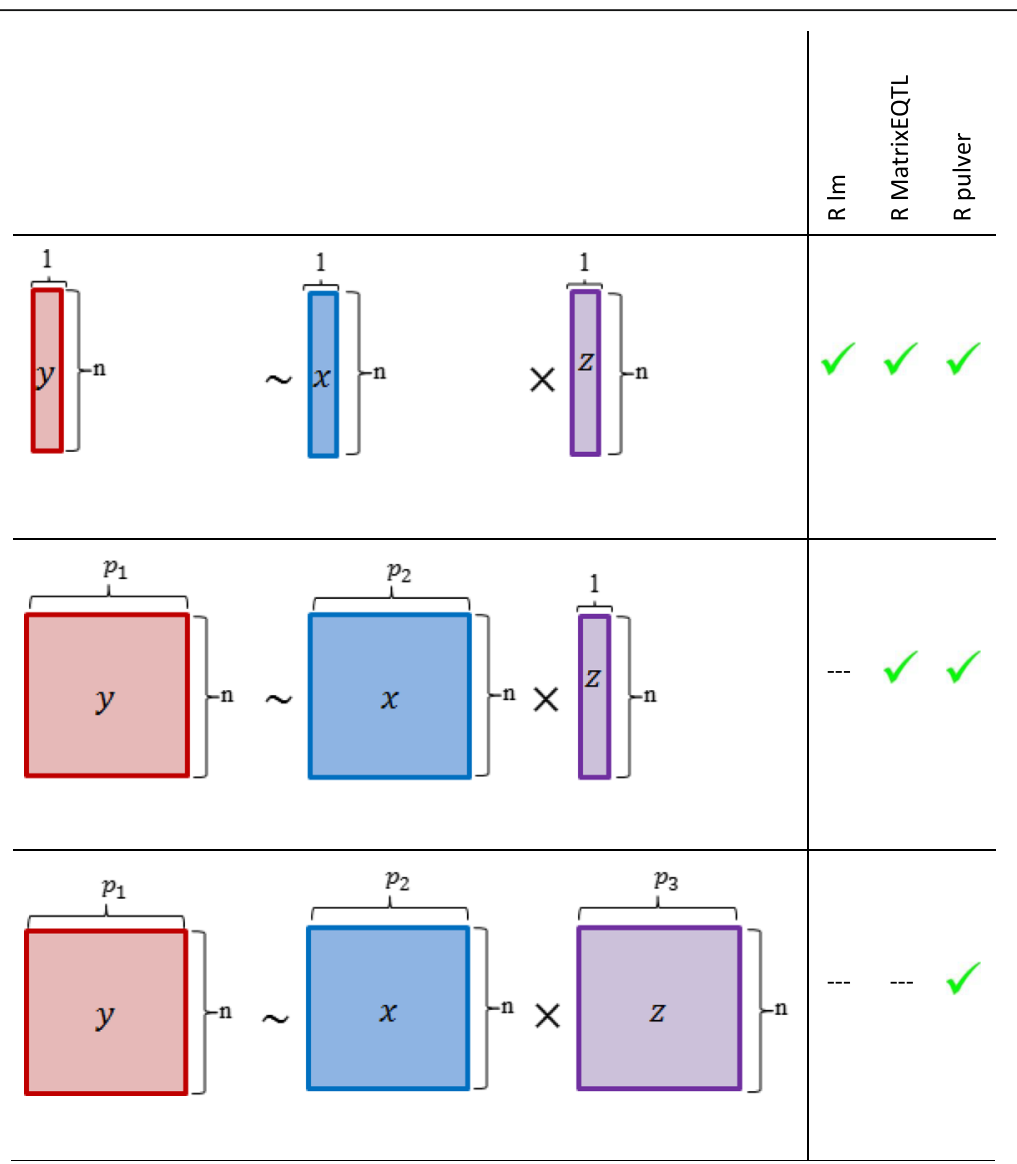

Fig. 2 Comparison of different input types handled by the R tools $I m$, MatrixEQTL, and pulver for computation of the linear regression with interaction term. By the braces the dimensions of the matrices are depicted. The R's build-in function Im can only compute the linear regression with interaction term using one variable with $n$ observations per call. The R package MatrixEQTL is able to compute simultaneously the linear regression for each of $p_{1}$ variables from the outcome matrix $Y$ and the interaction term of the matrix $X$ with $p_{2}$ variables and the vector $Z$. In contrast, pulver in addition iterates through $p_{3}$ variables of the matrix $Z$ and finally computes the linear regression for each column of matrices $Y, X$ and $Z$

in R's Im function or MatrixEQTL. However, parallelization is possible and it leads to significant speedups, although sublinear. For benchmarking purposes, each scenario was run 200 times using the $\mathrm{R}$ package microbenchmark (version 1.4-2.1, https://CRAN.R-project.org/package= microbenchmark) and the results were filtered with a $p$-value threshold of 0.05 .

Figure 3 shows that pulver performed better than the alternatives in all the benchmarks. Note that the benchmark results obtained for the $l m$ function were so slow that they could not be included in the chart.

In particular, for the benchmark where the number of variables in matrix $Z$ was varied (see Fig. $3 \mathrm{~d}$ ), pulver outperformed the other methods by several orders of magnitudes, and the results obtained by MatrixEQTL could not be included in the chart. The poor performance of MatrixEQTL is because it can only handle one $Z$ variable, which forced us to repeatedly call MatrixEQTL for every variable in the $Z$ matrix. This type of iteration is known to be slow in $\mathrm{R}$. The good performance of pulver with benchmark $\mathrm{d}$ is particularly notable because this benchmark reflects the intended user case for pulver where all input matrices contain many variables.

\section{Applying pulver to the analysis of real-world data}

Metabolites are small molecules in blood whose concentrations can reflect the health status of humans [19]. Therefore, it is useful to investigate the potential effects of genetic and epigenetic factors on the concentrations of metabolites.

DNA methylation denotes the attachment of a methyl group to a DNA base. Methylation occurs mostly on the cytosine nucleotides preceding a guanine nucleotide, which are also called cytosine-phosphate-guanine (CpG) sites [20]. DNA methylation was measured using the Illumina InfiniumHumanMethylation450 BeadChip platform, which quantifies the relative methylation of CpG sites [21]. 

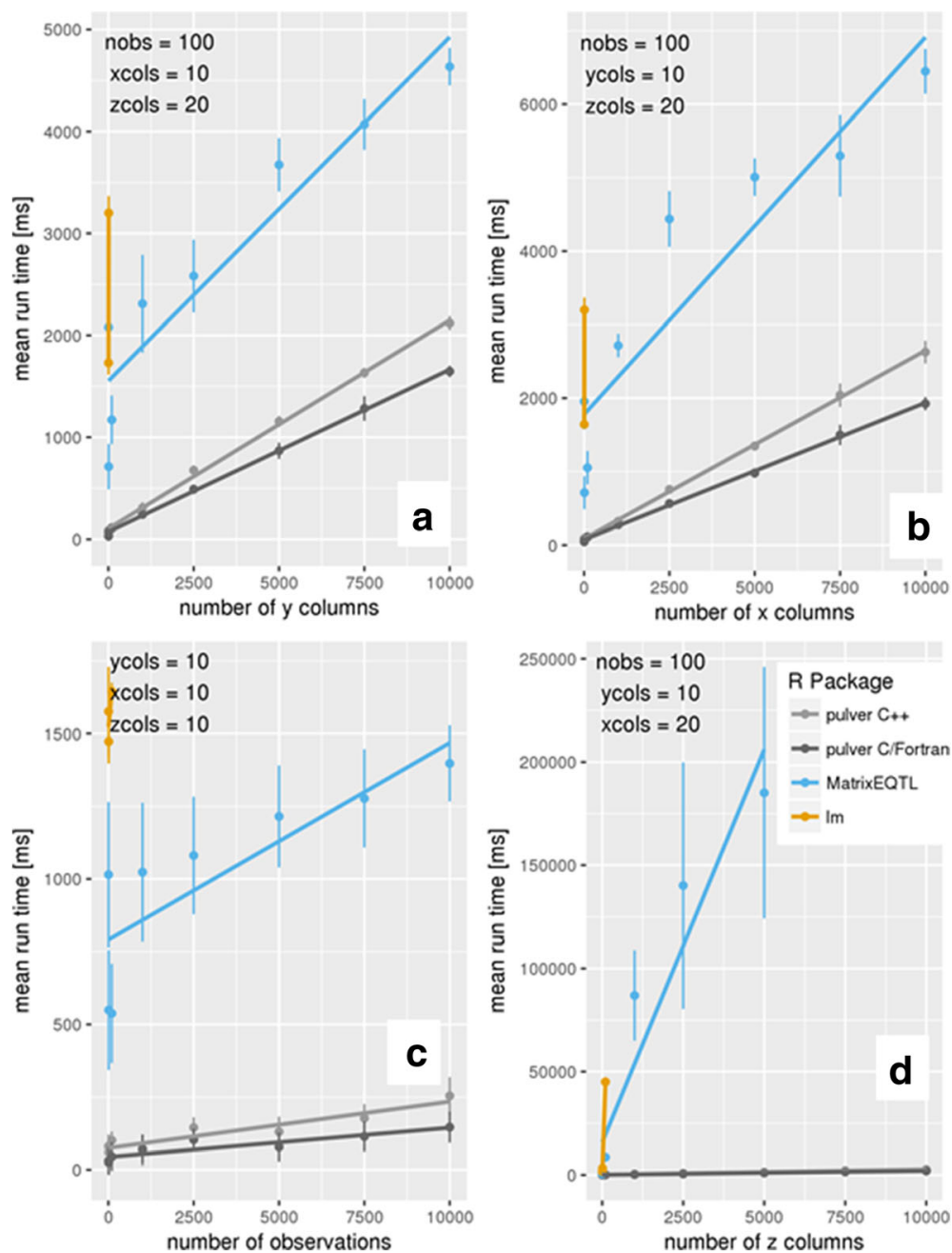

Fig. 3 Mean run times and standard deviations for interaction analysis using R's Im function, MatrixEQTL, and pulver. The execution times are in milliseconds. We fitted a line through the time points for each package. R's Im function was very inefficient for this type of interaction analysis, and only the first two points are shown for every benchmark. Shown are four different panels (a-d). In panel a the number of columns of the matrix is set to 10, the matrix to 20 and the number of observations is set to 100 , while the number of columns for the matrix is varied from 10 to 10,000 . In panel $\mathbf{b}$ number of columns of the matrix is varied from 10 to 10,000 while the number of columns for the matrix is set to 10 column, the matrix to 20 column and number of observations is set to 100 . In panel c the number of observations are varied from 10 to 10,000 while the number of columns for each matrix are fixed (all with 10 columns). In panel $\mathbf{d}$ number of columns of the matrix is varied from 10 to 10,000 , while the number of columns of the matrix is set to 20 , the matrix to 10 and the number of observations is set to 100

DNA methylation was measured in whole blood so it was based on a mixture of different cell types. We employed the method described by Houseman et al. [22] and adjusted for different proportions of cell types. Thus, CpG sites were represented by their residuals after regressing on age, sex, body mass index (BMI), Houseman variables, and the first 20 principal components of the principal component analysis control probes from $450 \mathrm{~K}$ Illumina arrays. The control probes were used to adjust for technical confounding, where they comprised the principal components from positive control probes, which were used as quality control for different data preparation and measurement steps.
Furthermore, to avoid false positives, all CpG sites listed by Chen et al. [23] as cross-reactive probes were removed. Cross-reactive probes bind to repetitive sequences or co-hybridize with alternate sequences that are highly homologous to the intended targets, which could lead to false signals.

In the KORA F4 study, genotyping was performed using the Affymetrix Axiom chip [24]. Genotyped SNPs were imputed with IMPUTE v2.3.0 using the 1000 Genomes reference panel.

Metabolite concentrations were measured using two different platforms: Biocrates (151 metabolites) and Metabolon (406 metabolites). Biocrates uses a kit-based, targeted 
quantitative by electrospray (liquid chromatography) - tandem mass spectrometry (ESI-(LC) MS/MS) method. A detailed description of the data was provided previously by Illig et al. [25]. Metabolon uses non-targeted, semiquantitative liquid chromatography coupled with tandem mass spectrometry (LC-MS/MS) and GC-MS methods. The data were previously described in Suhre et al. [26].

Metabolites were represented by their Box-Cox transformed residuals after regressing on age, sex, and BMI. We used the $\mathrm{R}$ package car [27] to compute the BoxCox transforms.

Initially, there were 345,372 CpG sites, 9,143,401 SNPs (coded as values between 0 and 2 according to an additive genetic model), and 557 metabolites in the dataset. Analyzing the complete data would have taken a very long time even with pulver.

Thus, to estimate the time required to analyze the whole dataset, we ran scenarios using all CpG sites, all metabolites, and different numbers of SNPs (100, 1000, 2000, 4000, and 5000), and extrapolated the runtime that would be required to analyze all SNPs. Due to time limitations, we ran each of the scenarios defined above only once. The estimated runtime required to analyze the complete dataset by parallelizing the work across 40 processors was 1.5 years.

Thus, we decided to only select SNPs that had previously known significant associations with at least one metabolite [1, 25]. We determined whether these signals became even stronger after adding an interaction effect between DNA methylation and SNPs.

To avoid an excessive number of false positives, the SNPs were also required to have a minor allele frequency greater than 0.05 . We applied these filters separately to the Biocrates and Metabolon data. After filtering, we had 345,372 CpG sites, 117 SNPs, and 16 metabolites for Biocrates, with 345,372 CpG sites, 6406 SNPs, and 376 metabolites for Metabolon.

We were only interested in associations that remained significant after adjusting for multiple testing, so we used a $p$-value threshold of $\frac{0.05}{345372^{*} 117^{*} 16+345372^{*} 6406^{*} 376}=6.01^{*}$ $10^{-14}$ according to Bonferroni correction.

We found 27 significant associations for metabolites from the Biocrates platform ( $p$-values ranging from $1.28^{*} 10^{-29}$ to $5.17^{*} 10^{-14}$ ) and 286 significant associations for metabolites from the Metabolon platform ( $p$-values ranging from
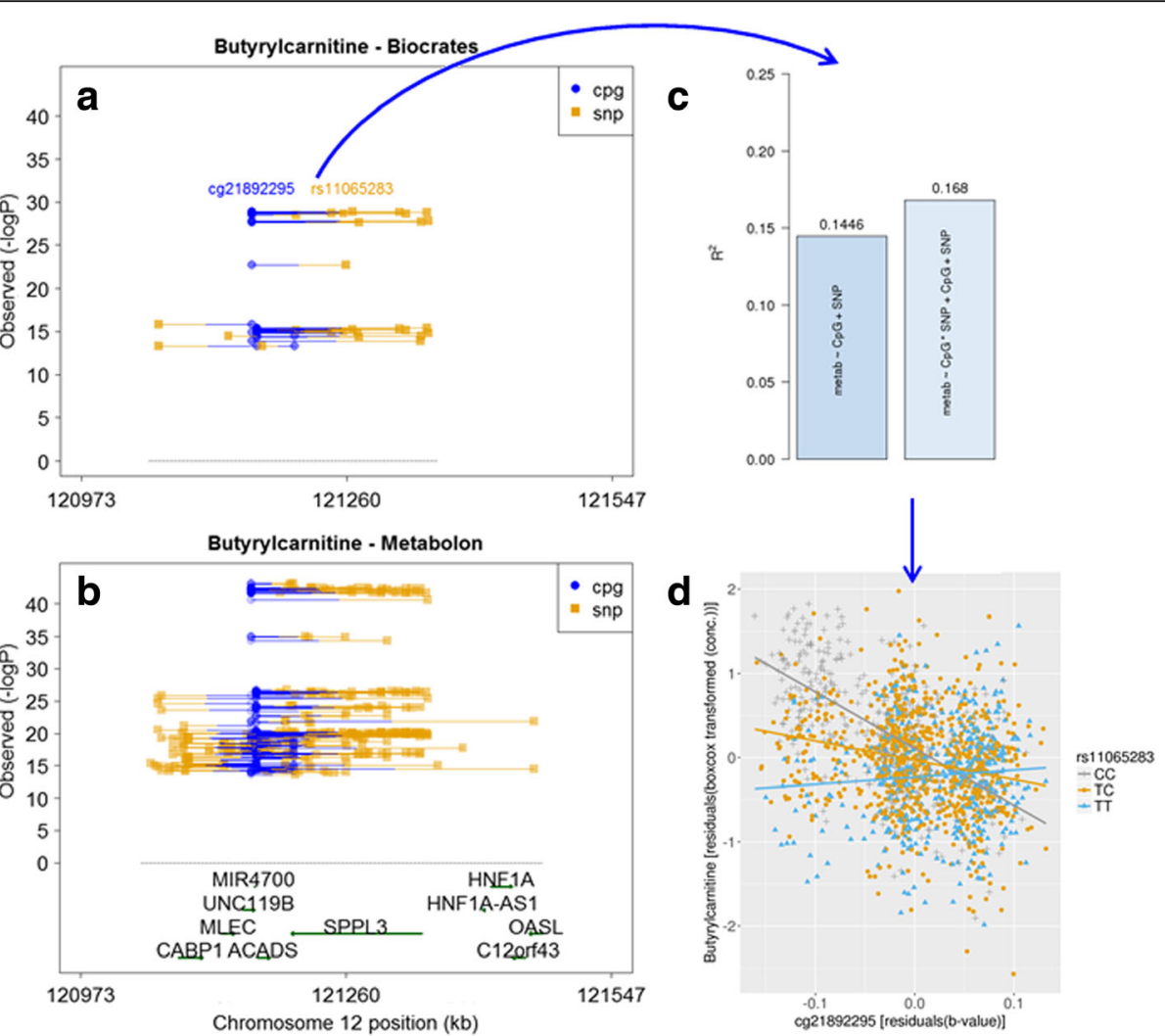

Fig. 4 Regional plot with significant associations among SNPs (circles), CpGs (squares), and butyrylcarnitine for the Biocrates platform (a) and Metabolon platform (b). Interactions between SNPs and CpGs are visualized by lines connecting SNPs and CpGs. c Comparison of the adjusted coefficient of determination in the models with and without the interaction term. $\mathbf{d}$ Scatterplot of CpG site cg21892295 and metabolite butyrylcarnitine. Genotypes are color-coded 
$1.15^{*} 10^{-42}$ to $3.73^{*} 10^{-14}$ ). All of the significant associations involved the metabolite butyrylcarnitine as well as SNPs and CpG sites on chromosome 12 in close proximity to the ACADS gene (see Fig. 4a and b). Figure 4c shows one of the significant results (SNP rs10840791, CpG site cg21892295, and metabolite butyrylcarnitine) to illustrate how the inclusion of an interaction term in the model increased the adjusted coefficient of determination, $\mathrm{R}^{2}$ (calculated using the summary.lm function in R).

The ACADS gene encodes the enzyme Acyl-CoA dehydrogenase, which uses butyrylcarnitine as a substrate [25], and previous studies have shown that SNPs and CpGs in this gene region are independently associated with butyrylcarnitine $[1,4,25]$.

\section{Discussion}

In the case where interaction terms need to be calculated for arbitrary pairs of variables, pulver performs far better than its competitors. The time savings are achieved by avoiding redundant calculations. Thus, computationally expensive $p$-values are only computed at the very end and only for results below a significance threshold determined using the (computationally cheap) Pearson's correlation coefficient. To maximize the speedup, we recommend always specifying a $p$-value threshold and using pulver as a filter to find models with significant or near-significant interaction terms. If a $p$ value threshold is not specified, the time savings will be suboptimal and the number of results will be very high.

Thus, we recommend using a $p$-value threshold to adjust for multiple testing, such as Bonferroni correction, i.e. $\frac{0.05}{\text { number of tests }}$, number of tests $=$ number of columns in $X \times$ number of columns in $Y \times$ number of columns in $Z$.

The core algorithm of pulver was implemented in two languages namely, $\mathrm{C}++$ and $\mathrm{C} /$ Fortran, to examine different performances due to programming languages. However, comparing the two different implementation of pulver reveals no striking differences. Thus, we continued to use the $\mathrm{C}++$ version as it offered some useful implemented functions such as those implemented in the C++ Standard Library algorithms [28].

The package imputes missing values based on their column means. If this is not required, then we recommend using other more sophisticated methods, such as the mice package in $\mathrm{R}$ [29], in order to remove missing values before applying pulver.

pulver was developed as a screening tool to efficiently identify associations between the outcome, such as metabolite levels, and the interaction among two quantitative variables, such as CpG-SNP interaction. Once, significant associations are identified, other information regarding the fitted models, such as slope coefficients, standard errors, or residuals, can be computed in a second step using traditional tools.

\section{Conclusion}

Our pulver package is currently the fastest implementation available for calculating $p$-values for the interaction term of two quantitative variables given a huge number of linear regression models. Pulver is part of a processing pipeline focused on interaction terms in linear regression models and its main value is allowing users to conduct comprehensive screenings that are beyond the capabilities of existing tools.

\section{Availability and requirements}

Project name: pulver.

Project home page: https://cran.r-project.org/web/packages/pulver/index.html

Operating system(s): Platform independent.

Programming language: $\mathrm{R}, \mathrm{C}++$.

Other requirements: $\mathrm{R}$ 3.3.0 or higher.

License: GNU GPL.

Any restrictions to use by non-academics: None.

\section{Additional file}

Additional file 1: Theory underlying pulver. This file describes the derivation of the t-value computed from the beta value divided by the standard error and the correlation value. (PDF $426 \mathrm{~kb}$ )

Abbreviations

GWAS: Genome-wide association studies; SNP: Single-nucleotide polymorphism

\section{Acknowledgements}

We thank all of the participants in the KORA F4 study, everyone involved with the generation of the data, and the two anonymous reviewers for comments.

\section{Funding}

The KORA study was initiated and financed by the Helmholtz Zentrum München - German Research Center for Environmental Health, which is funded by the German Federal Ministry of Education and Research (BMBF) and by the State of Bavaria. Furthermore, KORA research was supported within the Munich Center of Health Sciences (MC-Health), LudwigMaximilians-Universität, as part of LMUinnovativ.

\section{Availability of data and materials}

pulver can be downloaded from CRAN at https://cran.r-project.org/web/ packages/pulver/.

The data used in the simulations were generated by the create_input_files function found in testing.R.

\section{Authors' contributions}

SM and CG designed the study. SM and CB wrote the pulver software and conducted computational benchmarking. SM, CB, SW, MN, KS, RW, MW, TM JA, GK, KS, AP, HG, FJT, and CG contributed to the data acquisition or data analysis and interpretation of results. SM wrote the manuscript. SM, CB, SW, MN, KS, RW,MW, TM, JA, GK, KS, AP, HG, FJT, and CG contributed to the review, editing, and final approval of the manuscript.

Ethics approval and consent to participate

The KORA study was approved by the local ethics committee ("Bayerische Landesärztekammer", reference number: 06068). 
All KORA participants gave their signed informed consent.

\section{Consent for publication}

Not applicable

\section{Competing interests}

The authors declare that they have no competing interests.

\section{Publisher's Note}

Springer Nature remains neutral with regard to jurisdictional claims in published maps and institutional affiliations.

\section{Author details}

${ }^{1}$ Research Unit of Molecular Epidemiology, Helmholtz Zentrum München, Neuherberg, Germany. ${ }^{2}$ Institute of Epidemiology II, Helmholtz Zentrum München, Neuherberg, Germany. ${ }^{3}$ German Center for Diabetes Research (DZD), Neuherberg, Germany. ${ }^{4}$ Department of Medicine I, University Hospital Grosshadern, Ludwig-Maximilians-Universität, Munich, Germany. ${ }^{5}$ Institute of Genetic Epidemiology, Helmholtz Zentrum München, Neuherberg, Germany. ${ }^{6}$ Chair of Genetic Epidemiology, IBE, Faculty of Medicine, LMU Munich, Munich, Germany. ${ }^{7}$ DZHK (German Centre for Cardiovascular Research), Partner Site Munich Heart Alliance, Munich, Germany. ${ }^{8}$ Institute of Human Genetics, Helmholtz Zentrum München, Neuherberg, Germany. ${ }^{9}$ Institute of Human Genetics, Technische Universität München, Munich, Germany. ${ }^{10}$ Genome Analysis Center, Helmholtz Zentrum München, Neuherberg, Germany. ${ }^{11}$ Institute of Experimental Genetics, Technical University of Munich, Freising-Weihenstephan, Germany. ${ }^{12}$ Institute of Bioinformatics and Systems Biology, Helmholtz Zentrum München, Neuherberg, Germany. ${ }^{13}$ Department of Twins Research and Genetic Epidemiology, Kings College, London, UK. ${ }^{14}$ Department of Biophysics and Physiology, Weill Cornell Medical College in Qatar, Doha, Qatar. ${ }^{15}$ Institute of Computational Biology, Helmholtz Zentrum München, Neuherberg, Germany. ${ }^{16}$ Department of Mathematics, Technische Universitat München, Garching, Germany.

Received: 23 March 2017 Accepted: 20 September 2017

Published online: 29 September 2017

\section{References}

1. Shin SY, Fauman EB, Petersen AK, Krumsiek J, Santos R, Huang J, Arnold M, Erte I, Forgetta $V$, Yang TP, et al. An atlas of genetic influences on human blood metabolites. Nat Genet. 2014:46(6):543-50.

2. Kettunen J, Tukiainen T, Sarin AP, Ortega-Alonso A, Tikkanen E, Lyytikainen $L P$, Kangas AJ, Soininen P, Wurtz P, Silander K, et al. Genome-wide association study identifies multiple loci influencing human serum metabolite levels. Nat Genet. 2012;44(3):269-76.

3. Draisma HH, Pool R, Kobl M, Jansen R, Petersen A-K, Vaarhorst AA, Yet I, Haller T, Demirkan A, Esko T. Genome-wide association study identifies novel genetic variants contributing to variation in blood metabolite levels. Nat Commun. 2015:6

4. Petersen AK, Zeilinger S, Kastenmuller G, Romisch-MargI W, Brugger M, Peters A, Meisinger C, Strauch K, Hengstenberg C, Pagel P, et al. Epigenetics meets metabolomics: an epigenome-wide association study with blood serum metabolic traits. Hum Mol Genet. 2014;23(2):534-45.

5. Maturana E, Pineda S, Brand A, Steen K, Malats N. Toward the integration of Omics data in epidemiological studies: still a "long and winding road". Genet Epidemiol. 2016;40(7):558-69.

6. Heyn H, Sayols S, Moutinho C, Vidal E, Sanchez-Mut JV, Stefansson OA, Nadal E, Moran S, Eyfjord JE, Gonzalez-Suarez E. Linkage of DNA methylation quantitative trait loci to human cancer risk. Cell Rep. 2014;7(2):331-8.

7. Ma Y, Follis JL, Smith CE, Tanaka T, Manichaikul AW, Chu AY, Samieri C, Zhou X, Guan W, Wang L. Interaction of methylation-related genetic variants with circulating fatty acids on plasma lipids: a meta-analysis of 7 studies and methylation analysis of 3 studies in the Cohorts for Heart and Aging Research in Genomic Epidemiology consortium. Am J Clin Nutr. 2016;103(2):567-78.

8. Bell CG, Finer S, Lindgren CM, Wilson GA, Rakyan VK, Teschendorff AE, Akan P, Stupka E, Down TA, Prokopenko I, et al. Integrated genetic and epigenetic analysis identifies haplotype-specific methylation in the FTO type 2 diabetes and obesity susceptibility locus. PLoS One. 2010;5(11):e14040.

9. Krumsiek J, Bartel J, Theis FJ. Computational approaches for systems metabolomics. Curr Opin Biotechnol. 2016;39:198-206.
10. Gyenesei A, Moody J, Laiho A, Semple CA, Haley CS, Wei WH. BiForce Toolbox: powerful high-throughput computational analysis of gene-gene interactions in genome-wide association studies. Nucleic Acids Res. 2012; 40(Web Server issue):W628-32.

11. Lee S, Lozano A, Kambadur P, Xing EP. An Efficient Nonlinear Regression Approach for Genome-wide Detection of Marginal and Interacting Genetic Variations. J Comput Biol. 2016;23(5):372-89.

12. Hemani G, Theocharidis A, Wei W, Haley C. EpiGPU: exhaustive pairwise epistasis scans parallelized on consumer level graphics cards. Bioinformatics. 2011;27(11):1462-5.

13. Fabregat-Traver D, Sharapov S, Hayward C, Rudan I, Campbell H, Aulchenko Y, Bientinesi P. High-Performance Mixed Models Based Genome-Wide Association Analysis with omicABEL software. F1000Research. 2014:3:200.

14. Shabalin AA. Matrix eQTL: ultra fast eQTL analysis via large matrix operations. Bioinformatics. 2012;28(10):1353-8.

15. Wichmann H-E, Gieger C, Illig T, group MKs. KORA-gen-resource for population genetics, controls and a broad spectrum of disease phenotypes. Das Gesundheitswesen. 2005;67(S 01):26-30.

16. Team RC. R: A language and environment for statistical computing. Vienna: R Foundation for Statistical Computing. 2015. https://www.r-project.org/.

17. Eddelbuettel D, François R, Allaire J, Chambers J, Bates D, Ushey K. Rcpp: Seamless R and C++ integration. J Stat Softw. 2011:40(8):1-18.

18. OpenMP A: OpenMP Application Program Interface V3. 0. OpenMP Architecture Review Board 2008.

19. Kastenmüller G, Raffler J, Gieger C, Suhre K. Genetics of human metabolism: an update. Hum Mol Genet. 2015:24(R1):R93-R101.

20. Jones PA. Functions of DNA methylation: islands, start sites, gene bodies and beyond. Nat Rev Genet. 2012;13(7):484-92.

21. Bibikova M, Barnes B, Tsan C, Ho V, Klotzle B, Le JM, Delano D, Zhang L, Schroth GP, Gunderson KL, et al. High density DNA methylation array with single CpG site resolution. Genomics. 2011;98(4):288-95.

22. Houseman EA, Accomando WP, Koestler DC, Christensen BC, Marsit CJ, Nelson $\mathrm{HH}$, Wiencke JK, Kelsey KT. DNA methylation arrays as surrogate measures of cell mixture distribution. BMC Bioinformatics. 2012;13:86.

23. Chen YA, Lemire M, Choufani S, Butcher DT, Grafodatskaya D, Zanke BW, Gallinger S, Hudson TJ, Weksberg R. Discovery of cross-reactive probes and polymorphic CpGs in the Illumina Infinium HumanMethylation450 microarray. Epigenetics : official journal of the DNA Methylation Society. 2013;8(2):203-9.

24. Livshits G, Macgregor AJ, Gieger C, Malkin I, Moayyeri A, Grallert H, Emeny RT, Spector T, Kastenmüller G, Williams FM. An omics investigation into chronic widespread musculoskeletal pain reveals epiandrosterone sulfate as a potential biomarker. Pain. 2015;156(10):1845.

25. Illig T, Gieger C, Zhai G, Romisch-Margl W, Wang-Sattler R, Prehn C, Altmaier E, Kastenmuller G, Kato BS, Mewes HW, et al. A genome-wide perspective of genetic variation in human metabolism. Nat Genet. 2010;42(2):137-41.

26. Suhre K, Shin SY, Petersen AK, Mohney RP, Meredith D, Wagele B, Altmaier E, Deloukas P, Erdmann J, Grundberg E, et al. Human metabolic individuality in biomedical and pharmaceutical research. Nature. 2011:477(7362):54-60.

27. Fox J, Weisberg S: An R Companion to Applied Regression, Second edn: Sage; 2011.

28. Stroustrup B: Programming: principles and practice using $\mathrm{C}++$ : Pearson Education; 2014

29. Buuren S, Groothuis-Oudshoorn K: mice: Multivariate imputation by chained equations in R. J Stat Softw 2011, 45(3).

\section{Submit your next manuscript to BioMed Central and we will help you at every step:}

- We accept pre-submission inquiries

- Our selector tool helps you to find the most relevant journal

- We provide round the clock customer support

- Convenient online submission

- Thorough peer review

- Inclusion in PubMed and all major indexing services

- Maximum visibility for your research

Submit your manuscript at www.biomedcentral.com/submit
) Biomed Central 